\title{
La fonction argumentale au regard des noms d'artefact
}

\author{
Pierre-André Buvet \\ LDI-CNRS, Université Paris 13 \\ pierreandre.buvet@gmail.com
}

\begin{abstract}
Résumé. Nous discutons de l'élaboration d'un dictionnaire monolingue portant exclusivement sur les noms d'artefact du français et des conséquences théoriques pour l'étude de la fonction argumentale. Nous montrons comment l'étude systématique et exhaustive de ces substantifs, exigée par la description lexicographique, fait apparaître des propriétés remarquables qui conduisent à préciser certains aspects du modèle de données utilisé pour nos analyses linguistiques. Nous nous intéressons plus particulièrement aux noms composés pour établir comment leur structure interne explique leur combinatoire.Après avoir précisé quelles sont les propriétés des noms d'artefact et comment ils sont décrits dans le dictionnaire, nous discutons de la fonction argumentale en regard de la fonction prédicative puis nous étudions l'analyse de la structure interne des noms d'artefact.
\end{abstract}

\begin{abstract}
We discuss the elaboration of a monolingual dictionary concerning exclusively the names of artefact of French and the theoretical consequences for the study of the argument function. We show how the systematic and exhaustive study of these nouns, required by the lexicographical description, reveals remarkable properties which lead to specify certain aspects of the model of data used for our linguistic analyses.
\end{abstract}

\section{$1 \quad$ Introduction}

Nous avons pour objectif ici d'établir en quoi l'analyse de la structure interne des noms argumentaux est révélatrice de leur mode de fonctionnement. Nous nous appuyons sur leur description lexicographique, car nous considérons qu'il s'agit d'une source de questionnement sur le fonctionnement des langues. Aussi, nous discutons de l'élaboration d'un dictionnaire monolingue portant exclusivement sur les noms d'artefact du français et des conséquences théoriques pour l'étude de la fonction argumentale. Nous montrons comment l'étude systématique et exhaustive de ces substantifs, exigée par la description lexicographique, fait apparaître des propriétés remarquables qui conduisent à préciser certains aspects du modèle de données utilisé pour nos analyses linguistiques. Nous nous intéressons plus particulièrement aux noms composés pour établir comment leur structure interne explique leur combinatoire. 
Après avoir précisé quelles sont les propriétés des noms d'artefact et comment ils sont décrits dans le dictionnaire, nous discutons de la fonction argumentale en regard de la fonction prédicative puis nous étudions l'analyse de la structure interne des noms d'artefact.

\section{Les noms d'artefact}

Les noms d'artefact sont des noms argumentaux du fait qu'ils sont incompatibles avec la fonction prédicative, $c f$. infra. Pour autant, la nature des objets qu'ils dénotent impliquant une finalité fonctionnelle, une partie d'entre eux spécifient explicitement une prédication car, lorsqu'il s'agit de mots construits, ils ont trait à des prédicats nominaux ou verbaux (arrosage/arroser $\rightarrow$ arrosoir) et, lorsque ce sont des mots simples, ils peuvent donner lieu à des prédicats nominaux ou verbaux (marteau $\rightarrow$ martellement/marteler). Par ailleurs, ils se conçoivent comme des holonymes (voiture) ou des méronymes (roue) tels que les relations partie-tout sont fondées sur des prédications spécifiques (le méronyme roue est ainsi défini par rapport à son holonyme : « organe.... Qui permet à un véhicule de reposer sur le sol et de s'y mouvoir » dictionnaire Larousse en ligne) $)^{1}$.

Pour le français, plus de 30000 noms d'artefact ont été recensés ${ }^{2}$. Nous discutons de leurs principales caractéristiques, sur les plans morphosyntaxique et sémantique, puis nous présentons le dictionnaire électronique qui les décrit.

\subsection{Propriétés morphologiques}

La liste des noms d'artefact du français est constituée de mots simples et de mots construits. Les premiers représentent $9 \%$ des substantifs recensés, par exemple couteau. Ce sont des " signes élémentaires " dans la mesure où ils " ne peuvent pas être décomposés en signes plus simples dont ils seraient constitués " POLGUERE 2003. Certains sont des emprunts, comme string qui est un mot d'origine anglaise.

Les mots construits sont principalement des noms dérivés (hachoir) ou des noms composés (minuterie automatique). Les premiers représentent $41 \%$ des substantifs recensés et les seconds $49 \%$. Les autres mots construits sont des abréviations comme $A P N$, dont la source est appareil photo numérique ou des apocopes comme ampli dont la source est amplificateur.

La grande majorité des dérivés sont des noms suffixés. Les différents types de suffixés correspondant à des noms d'artefact sont distinguables selon la nature grammaticale de leur base (DUBOIS 1962) :

- $\quad$ base adjectivale : chaudière

- $\quad$ base nominale : auget, aviateur, bobinot, boiserie, bonbonnière, bordelaise, bordure, bottillon, bruiteur, cordage, cuissarde, drageoir, goulotte, moutardier, serpette,

- base verbale : accotement, alliance, accoudoir, affûteuse, alternateur, barrière, bouillotte, bouterolle, échafaudage, fixation, gicleur, imprimante

\footnotetext{
${ }^{1}$ Dans le cadre de la théorie sens-texte, la prédicativité dont font état les noms argumentaux conduit à les catégoriser comme des quasi-prédicats sémantiques. C'est-à-dire des lexies qui ont « un sens liant » et qui « dénotent un fait » POLGUERE 2012.

${ }^{2}$ La macrostructure du dictionnaire découle de la concétanation de listes terminologiques et d'ontologies ainsi que de l'analyse de corpus spécialisés. La définition de la macrostructure du dictionnaire s’inscrit dans la continuité d'autres travaux sur les ressources linguistiques dédiées au traitement de l'information textuelle [BUVET 20\$\$].
} 
Il existe également quelques cas de dérivation zéro, appelée également conversion ; par exemple, le nom applique. Les composés recensés sont d'une grande variété. Ils sont étudiés plus en détail dans la section 4.

\subsection{Propriétés sémantiques}

La notion d'artefact est définie comme suit dans les dictionnaires :

- 'produit de l'art ou de l'industrie humaine' (Grand Robert de Langue Française)

- 'produit ayant subi une transformation, même minime, par l'homme, et qui se distingue ainsi d'un autre provoqué par un phénomène naturel' (Larousse).

- 'produit de l'art, de l'industrie, objet artificiel' (TLF)

Les noms d'artefact ont des particularités linguistiques remarquables (CORBIN \& CORBIN 1991). Ils correspondent à des holonymes, comme lave-linge, ou bien des méronymes, comme couvercle. Les noms de la première catégorie dénotent des ensembles constitués d'éléments et ceux de la seconde catégorie les éléments de ces ensembles. Le terme de relation partie-tout est souvent employé pour désigner le mode d'agencement des éléments au sein d'un ensemble (KLEIBER \& Alii 2006). Les noms d'holonyme recensés sont des noms d'appareil, par exemple clouteuse, des noms de cosmétique, par exemple rouge à lèvres, des noms d'instrument, par exemple guitare, des noms de machine, par exemple $p c$, des noms de moyens de transport, par exemple camping-car et des noms de vêtement, par exemple chemise. Les noms de méronyme recensés sont notamment des noms de dispositif, par exemple écouteur, des noms d'organe, par exemple embout, des noms d'ouverture, par exemple regard, des noms de partie générique, par exemple face intérieure, et des noms de partie de vêtement, par exemple col.

Certains substantifs se comportent comme un holonyme ou comme un méronyme selon les contextes. C'est le cas de noms de contenant ou d'ustensile. Ainsi, pince fonctionne comme un holonyme dans Il a utilisé la pince pour serrer le boulon et un méronyme dans Le robot s'est avancé d'un mètre puis a dirigé sa pince vers le boulon. De même, bol fonctionne comme un holonyme dans J'ai versé le café dans le bol et un méronyme dans J'ai un mixeur mais le bol est fendu.

Les noms d’artefact appartiennent aussi bien à la langue générale, par exemple serrure, qu’aux langues spécialisées, par exemple tube à essai. Dans le second cas de figure, les approches terminologiques sont souvent mobilisées pour analyser ces substantifs car ils ont une double dimension: linguistique et extralinguistique (LERAT 1995). Il existe toutes sortes de nomenclatures et de taxinomies décrivant les noms d'artefact, qu'il s'agisse d'holonymes ou de méronymes (SLIWA 2000). Les noms d'artefact sont par ailleurs attestés depuis des siècles, par exemple merlin ou au contraire sont des créations récentes, par exemple zapette. Enfin, ils appartiennent à différents registres; ainsi gapette appartient à la langue familière tandis que couvre-chef est d'un niveau de langue plutôt soutenu.

Les noms d’artefact dénotent des objets dont la création est conditionnée par une fonctionnalité précise. De ce fait, ils sont associés à des adjectifs, des noms ou des verbes en rapport avec cette fonctionnalité. Ces associations constituent un savoir linguistique des locuteurs se manifestant par des énoncés préconstruits. Par exemple, couteau est un nom d'artefact associé à l'adjectif tranché et aux verbes couper et découper. Les énoncés préconstruits sont des phrases génériques reposant sur des stéréotypes (ANSCOMBRE 2009), (FRADIN 1984) et (KLEIBER 1990) : Un couteau, c'est tranchant ; Un couteau, ça coupe ; Un couteau, ça découpe.

L'analyse de la structure interne des noms d'artefact explique souvent ces associations sémantiques lorsqu'il s'agit de mots construits. C’est notamment le cas des noms suffixés formés à parti d'une base 
verbale. Les verbes constitutifs des suffixés ont des synonymes, des hyperonymes ou des hyponymes qui sont impliqués dans les énoncés préconstruits associés aux noms d'artefact. Ainsi, le nom mixeur est formé sur mixer défini sémantiquement par la classe MELANGE. Cette classe définit également des noms comme mélange et des verbes comme brasser, mélanger, mixer, remuer que l’on trouve dans les énoncés préconstruits associés à mixeur : Un mixeur, ça brasse ; Un mixeur, ça fait des mélanges ; Un mixeur, ça mélange ; Un mixeur, ça mixe ; Un mixeur, ça remue.

\subsection{Description lexicographique}

Les noms d'artefact sont recensés et décrits dans un dictionnaire électronique ${ }^{1}$ dont la nomenclature est constituée des noms argumentaux. Ces substantifs ne fonctionnent jamais comme des prédicats en discours ; il peut s’agir de noms simples (alambic) ou de noms construits du type suffixé (boitier) ou du type composé (boite de vitesse), cf. supra. Ils constituent la macrostructure du dictionnaire. Les variations de forme d'un nom donné constituent autant d'entrées du dictionnaire ; bracelet cuir et bracelet en cuir sont deux entrées différentes.

La microstructure est constituée de la vedette et de cinq descripteurs : 1) l'hyperclasse ; 2) la classe ; 3) domaine 1 ; 4) domaine2 ; 5) domaine 3 :

poignée de porte/,.N/H_ORGANE/C_ORGANE_PREHENSION/D1_HABITATION/D2_TRAVAUX_E T_EQUIPEMENTS_MENAGERS/D3_TECHNIQUES

Les deux premiers descripteurs sont de nature sémantique. Le descripteur 'hyperclasse' concerne 18 catégories d'artefact: ALIMENT (yaourt); APPAREIL (bouveteuse); CONTENANT (bassine); COSMETIQUE (lait de maquillage); DISPOSITIF (barrière de protection); DOCUMENT (plaque d'identité); GENERIQUE (produit); INSTRUMENT (guitare); MACHINE (tablette tactile); MOYEN_TRANSPORT (bicyclette) ; ORGANE (bouton) ; OUVERTURE (passage de roue avant) ; PARTIE_GENERIQUE (Sommet) ; PARTIE_VETEMENT (Manche) ; REVETEMENT (Plancher) ; SUPPORT (fiche); USTENSILE (Couteau) ; VETEMENT (veste).

Le descripteur 'classe 'rassemble des catégories dénommées selon le principe suivant :

'NOM D’HYPERCLASSE’_NOM DE FONCTION D’ARTEFACT’.

C'est ainsi que l'hyperclasse OUVERTURE subsume les classes suivantes : OUVERTURE_CONNEXION (orifice de compensation); OUVERTURE_DIFFUSION (orifice de vaporisation) ; OUVERTURE_EVACUATION (évent à vapeur) ; OUVERTURE_FIXATION (œillet) ; OUVERTURE_GENERIQUE (ouverture); OUVERTURE_PASSAGE (bouche d'égout); OUVERTURE_TRANSMISSION (trou de serrure).

Près de 150 noms de fonction sont associés à des noms d'artefact de telle sorte que le descripteur classe est formé d’environ 370 items.

Les trois derniers descripteurs sont de nature pragmatique. Ils portent tous sur le domaine d'emploi des noms d'artefact. Le domaine d'emploi est le secteur d'activité relatif à l'usage d'un mot : « les indications de domaine n'indiquent pas seulement le champ d'expérience dont relève le mot [...] mais aussi à propos duquel on l'utilise » QUEMADA 1984.Seul le premier descripteur est obligatoirement spécifié, les deux autres le sont si le nom d'artefact relève de plus d'un domaine. 


\section{Modèle de données}

La théorie des trois fonctions primaires est le modèle de données auquel nous nous référons (HAJOK \& MEJRI 2011) et (BUVET 2013). Nous présentons brièvement la théorie puis nous discutons de la spécificité de l'une des rois fonctions, la fonction argumentale, et nous montrons comment les noms d’artefact illustrent cette spécificité.

\subsection{Les trois fonctions primaires}

La théorie des trois fonctions primaires s'inscrit dans la lignée des théories qui ont comme point de départ les analyses linguistiques de Zellig S. Harris (HARRIS 1976) et s’inspire également des travaux de Robert Martin (MARTIN 2014). Elle postule que les notions d'argument et de prédicat sont d'origine logico-sémantique, les arguments se rapportant à des entités et les prédicats à leurs attributs (prédicats monadiques) et aux relations qui les caractérisent (prédicats diadyques, triadyques, ...) pour donner lieu à des représentations langagières de l'univers. La notion de modalisation implique que l'énonciateur est consubstantiel à l'énoncé, c.-à-d. ce qu'il énonce est inscrit dans son point de vue, point de vue qui implique également le temps et l'espace de l'énonciation (BENVENISTE 1966).

La théorie a pour finalité d'expliquer les mécanismes langagiers en privilégiant le lexique comme objet d'étude. Il s'agit d'analyser conjointement les propriétés morphologiques, syntaxiques et sémantiques des unités linguistiques selon qu'elles fonctionnent comme des prédicats (fonction prédicative), des arguments (fonction argumentale) ou des modalisateurs (fonction modalisatrice).

Les structures prédicat-argument sont conçues comme autant d'éléments fondamentaux d'une composante d'un savoir linguistique partagé. Elles permettent de formuler des contenus propositionnels. L'actualisation est le moyen d'instancier les structures prédicat-argument dans le discours en produisant des énoncés bien formés relativement à des situations d'énonciation particulières. Elle s'explique notamment en termes de fonction modalisatrice dans la mesure où elle fait appel à des catégories énonciatives comme la personne, le temps, l'aspect, l'intensité, etc. De ce point de vue, elle est imputable aux actualisateurs qui permettent l'ancrage des prédicats et de leurs arguments dans un discours donné en fonction de la position du locuteur par rapport à ce qu'il énonce. Les actualisateurs, tant du point de vue de leur forme que de celui de leur combinatoire avec les structures prédicat-argument, constituent une autre composante de savoir linguistique : la grammaire de la langue considérée.

\subsection{La fonction argumentale}

Le modèle de données élaboré jusqu'à présent a surtout privilégié la fonction prédicative au détriment de la fonction argumentale. L'analyse des arguments les fait dépendre des prédicats, les seconds souscatégorisant les premiers de telle sorte que les propriétés sémantiques des arguments sont toujours rapportées aux prédicats. Le concept de classes d'objets illustre cette analyse en corrélant le fonctionnement sémantique des arguments à leur combinatoire avec des prédicats dits appropriés (MATHIEU-COLAS et LE PESANT 1998). Néanmoins, l'inclusion mutuelle, la présupposition d'existence réciproque et la complémentarité des trois fonctions primaires ne justifie pas de mettre en avant la fonction prédicative au détriment des deux autres. Nous le démontrons ici à propos de la fonction argumentale

Les arguments des prédicats sont instanciés dans un énoncé par le biais des constructions suivantes : des groupes nominaux, des complétives et des infinitives. Ces constructions, quel que soit leur type, occupent les positions sujet et complément dans les constructions qui caractérisent les emplois prédicatifs : la 
voiture et la route dans La voiture roulait sur la route; Qu'il fasse beau dans Qu'il fasse beau est surprenant; Mettre une cravate dans Mettre une cravate n'impressionne plus autant. La nature phrastique des arguments est inhérente aux constructions du type complétive ou du type infinitive (LE GOFFIC 1993). Pour les groupes nominaux, on distingue les arguments qui sont intrinsèquement de nature phrastique de ceux qui ne le sont pas. Dans le premier cas de figure, le groupe nominal s'analyse comme une phrase nominalisée (GUIRAUD 1967); par exemple, le refus de Léa dans Le refus de Léa était inattendu. La substitution du groupe nominal par une complétive ou une infinitive sémantiquement équivalentes atteste de la nature phrastique de l'argument: Que Léa ait refusé était inattendu ; Avoir refusé était inattendu de la part de Léa.

Dans le deuxième cas de figure, le groupe nominal n'est pas la nominalisation d'une phrase instanciée car la tête ne correspond jamais à un prédicat sous une forme nominale ; par exemple, dans La voiture de Luc a eu un accident, le groupe nominal La voiture de Luc s'analyse comme la réduction d'une phrase formée à partir d'un prédicat dyadique dont seuls les deux arguments sont présents dans le groupe nominal (Luc a acheté une voiture, Luc conduit une voiture, ...). Lorsque les réductions d'une phrase en groupe nominal ne sont pas des nominalisations, le groupe nominal n'est pas substituable à une complétive ou à une infinitive sémantiquement équivalente.

Ces analyses des groupes nominaux conduisent à distinguer deux catégories de substantifs : les noms prédicatifs (refus) et les noms argumentaux (voiture). Les premiers correspondent à des prédicats instanciés sous une forme nominale, mais ce n’est jamais le cas des seconds. Les noms prédicatifs sont compatibles aussi bien avec la fonction prédicative (Léa a opposé un refus) qu'avec la fonction argumentale (Le refus de Léa était inattendu). Les noms argumentaux sont compatibles uniquement avec la fonction argumentale ( $\mathrm{La}$ voiture de Luc est en panne). Lorsque les groupes nominaux sont formés d'un prédéterminant et d'un nom, la catégorisation reste pertinente car les noms prédicatifs sont substituables avec des constructions à support (DALADIER 1996); On parle d'un refus/On parle d'opposer un refus. Les noms argumentaux sont réfractaires à de telles substitutions. Dans ce qui suit, seuls les noms argumentaux sont pris en compte car ils sont les plus représentatifs de la fonction argumentale. Cette opposition fait écho à celle des noms catégorématiques (voiture) et des noms syncatégorématiques (refus), selon laquelle la signification des noms syncatégorématiques, contrairement à celle des noms catégorématiques, n’est pas immédiatement accessible (KLEIBER 1981).

La dénomination et la définition sont deux concepts fondamentaux pour l'analyse des substantifs. Elles portent respectivement sur deux aspects constitutifs des mots, leur forme et leur valeur. La dénomination est centrée sur le premier aspect et la définition sur le second.

Rappelons que la dénomination est « la relation qu’établit un locuteur (individuel ou collectif) entre luimême et son public en attribuant un nom à un segment de réalité, dont l'existence est plus ou moins préalablement admise » MORTUREUX 1984. Cette relation «parce qu'elle unit un nom à un concept rassembleur d'occurrences particulières, permet, une fois la compétence acquise, d'utiliser le nom en question, sans nouvel apprentissage, pour tout particulier qui présente les propriétés spécifiques de la classe délimitée par le concept en question » KLEIBER 1984. La dénomination participe de l'ontologie car elle contribue à spécifier l'ensemble des objets propres à un domaine donné (PETIT 2009). De ce point de vue, les noms argumentaux, en tant que noms catégorématiques, par le seul biais de la dénomination, donnent lieu à la catégorisation des entités qu'elles soient concrètes, animaux, artefacts, minéraux, locatifs, végétaux, etc., ou bien abstraites, par exemple la taxinomie des disciplines scientifiques.

La définition est complémentaire de la dénomination. Elle permet de traiter les mots en les associant à des descripteurs sémantiques, plus ou moins normalisés, afin d’expliciter leur valeur. La pratique 
lexicographique, a systématisé l’usage des définitions en mettant en avant deux relations lexicales $\left(\right.$ PRUVOST 2006) ${ }^{2}$ :

1. la relation hyponymie/hyperonymie ; par exemple, le début de la définition du mot cheval est :

- 'mammifère domestique’ dans le Trésor de la Langue Française Informatisé ;

- 'grand mammifère' dans le Grand Robert de la Langue Française ;

- 'mammifère herbivore' dans le Larousse en ligne;

2. la relation holonymie/mérononymie ; par exemple, le début de la définition du mot museau est :

- ' 'partie antérieure’ dans les trois dictionnaires.

Le concept de genre prochain, d'inspiration aristotélicienne, est fondamental en métalexicographie (REYT-DEBOVE 1978) ; il est fondé sur les deux relations lexicales mentionnées. Mais, la définition d'un mot ne se limite pas au genre prochain, elle comporte également la différence spécifique (IMBS 1960). Parmi les éléments qui contribuent à la préciser, il y a des informations faisant état de la finalité de l'entité dénommée dans l'entrée; musée, par rapport au genre proche spécifié par 'lieu' ou ‘établissement', a comme différence spécifique :

- 'où sont conservés, répertoriés, classés des objets, des documents, des collections d'intérêt artistique, scientifique ou technique’ dans le Trésor de La Langue Française Informatisée.,

- 'dans lequel sont rassemblées et classées des collections d'objets présentant un intérêt historique, technique, scientifique, et, spécialement, artistique, en vue de leur conservation et de leur présentation au public’ dans le Grand Robert de la Langue Française et

- ' 'où sont réunies, en vue de leur conservation et de leur présentation au public, des collections d'œuvres d'art, de biens culturels, scientifiques ou techniques’ dans le Larousse en ligne.

Il en résulte que toutes sortes d'énoncés préconstruits sont formulables à partir des dénominations : Dans un musée, on conserve des objets ; Dans un musée, on répertorie des objets ; Dans un musée, on présente des objets ; etc.

La dénomination des noms argumentaux contribuant à la réalisation d'ontologie et leur définition étant à la source des discours qui s'appuient sur leur dénomination, la combinatoire des arguments s'explique à partir de leur sémantisme propre (HOEY 2006). Pour les noms argumentaux, la juxtaposition de l'approche dénominative et de l'approche définitionnelle a le mérite de synthétiser les analyses ontologiques, qui rendent ces substantifs directement accessibles sur le plan conceptuel à partir de leur statut référentiel, et les analyses syntactico-sémantiques qui les catégorisent à partir de de leurs distributions remarquables (celles qui sont sous-jacentes aux définitions).

Ces différents points conduisent à s’interroger sur le rôle des noms argumentaux dans l'étude des faits de langue. Etant détenteurs d'énoncés préconstruits accessibles par leur mode de classification et de définition, le statut de la fonction argumentale par rapport à celui des autres fonctions primaires, et notamment la fonction prédicative, serait donc à reconsidérer car elle aurait aussi un rôle de premier plan dans le modèle de données. Cette analyse est corroborée par l'étude des artefacts. 


\subsection{Des noms argumentaux remarquables}

Les noms d'artefact constituent un observatoire privilégié pour la fonction argumentale du fait que leur dénomination se prête facilement à la constitution d'ontologie (les noms de l'audiovisuel, de l'électroménager, de la marine, etc.) et que leur définition permet, d'une part, de les associer à des hyperonymes (appareil, moyen de transport, ustensile,...) ou des méronymes (organe, ouverture, partie, ...) et, d'autre part, de les associer à une classe sémantique en rapport avec leur fonctionnalité.

Dans les cas de dérivation nominale, le substantif peut servir de base à un nom prédicatif en rapport avec sa fonctionnalité (bâche -> bâcher et bâchage). Il peut aussi être est formé à partir d'une racine prédicative faisant état de cette fonctionnalité (balayer -> balayeuse). De nombreux travaux en morphologie ont montré comment dériver la signification globale des noms d'artefact à partir de celle de leurs formants Dans les cas de composition savante, la signification des formants, d'origine latine ou grecque, rend explicite la fonctionnalité de l'artefact (bolomètre est formé du formant grec bolo- qui signifie 'radiation' et de du formant grec -mètre qui signifie 'mesure', un bolomètre étant défini comme un 'détecteur de rayonnement électromagnétique') (MITTERAND 1963). Dans les cas de composition non savante, la fonctionnalité de l'artefact est parfois déductible de l'un des constituants ; par exemple poêle à frire signifie 'ustensile de cuisine pour faire la friture', $c f$. infra.

Il existe des contre-exemples, par exemple l'analyse de la structure interne de baladeur ne permet pas d'établir que sa définition est 'appareil portable diffusant des données sonores'. Par ailleurs, tous les noms d'artefact ne possèdent pas une structure interne analysable. Par exemple la définition de canne : 'ustensile qui permet de marcher ou de se soutenir', n'est pas directement déductible à partir de de sa dénomination. Pour autant, la définition des noms d'artefact, quel qu'ils soient, fonctionne remarquablement sur le principe du genre proche et de la différence spécifique, cette dernière étant révélatrice des énoncés préconstruits constitutifs de ces substantifs: Avec une canne, on marche; Avec une canne, on s'appuie. De ce point de vue, les noms d'artefact sont bien des noms argumentaux exemplaires car leurs propriétés morphosémantiques permettent d'expliquer les discours qui les incorporent.

Pour ce qui est du dernier point, les propriétés de la structure interne des noms argumentaux déterminent souvent les propriétés de leur structure externe. Qu'il s'agisse d'unités monolexicales ou polylexicales, leur combinatoire est souvent prédictible à partir de leurs propriétés morphologiques. Après l'avoir observé rapidement pour les noms suffixés, nous allons le constater d'une façon plus approfondie pour les noms composés.

\section{Analyse de la structure interne des noms composés ${ }^{3}$}

\subsection{Diversité taxinomique}

Les noms d'artefact se prêtent à plusieurs classifications. Du point de vue morphologique, on observe une grande diversité de configurations. Ils se distinguent selon qu'ils comportent :

- $\quad$ uniquement des unités monolexicales mots simples : par exemple, feu de recul ;

- $\quad$ au moins une unité polylexicale : par exemple, feu rouge arrière.

Dans le premier cas de figure, parmi les différentes configurations possibles il y a notamment :

- ADJECTIF+NOM (grande tourisme, haut-fourneau, petite cuillère) ;

- NOM+ADJECTIF (ampoule torsadée, bouton rotatif, disque tournant) ;

- NOM+NOM (appareil inhalateur, émetteur USB, meuble télé) 
- NOM+DETERMINANT+NOM (coffret dix voitures, commutateur huit positions, vis-sixpans, )

- NOM+PREPOSITION+NOM (ampoule-à-culot, bouton de verrouillage, assiette en carton) ;

- NOM+PREPOSITION+DETERMINANT+ NOM (banquette de la voie, cadran des températures, dé à huit faces)

- NOM+PREPOSITION+VERBE (aiguille à brider, grattoir à déjointer, machine à laver, ) ;

- VERBE+NOM (garde-manger, gratte-ongles, vide-poussière)

Bien qu'elles soient moins fréquentes, d'autres configurations sont également possibles, par exemple VERBE+PRONOM (fourre-tout)

Dans le second cas de figure, la diversité configurationnelle est encore plus marquée :

- NOM+VERBE+NOM (bonde pare-bruit)

- NOM+PREPOSITION+NOM+ADJECTIF (bouton en verre calorifuge)

- NOM+PREPOSITION+NOM+PREPOSITION+NOM (émetteur de rayonnement à infrarouge)

- NOM+ADJECTIF+PREPOSITION+DETERMINANT+NOM (crème lavante pour les mains)

- VERBE+NOM+ADJECTIF (grille-pain électrique)

- etc.

Il est cependant possible de rapporter ces configurations à celles du premier cas de figure, les mots complexes partageant les mêmes catégories grammaticales que les mots simples. Par exemple, la construction du substantif témoin de température du liquide de refroidissement est du type NOM+PREPOSITION+DETERMINANT+NOM dans la mesure où les séquences témoin de température et liquide de refroidissement correspondent à des noms. Il arrive qu'une même configuration se rapporte à deux autres. Par exemple, NOM+PREPOSITION+NOM+ADJECTIF est rapportable au type NOM+PREPOSITION+NOM lorsqu'il s'agit de la séquence joint de culasse électronique et au type NOM ADJECTIF avec la séquence interrupteur de poste télécommandé.

Sur le plan morphosyntaxique, les noms composés sont distingués selon qu'ils fonctionnent de façon endocentrique ou de façon exocentrique (GONZÁLEZ REY 2002). Les premiers sont réductibles à leur tête nominale, qu'elle soit simple, accessoire de jeu => accessoire, ou complexe, suspension arrière à roues indépendantes $=>$ suspension arrière ${ }^{4}$. Les seconds ne le sont pas soit parce qu'ils ne comportent pas de noms en position frontale, par exemple fume-cigarette, soit parce que le substantif initial n'est pas une tête nominale, par exemple pointe-de-cœur ${ }^{5}$.

Les noms composés se différencient également selon leur caractère sémantiquement transparent ou opaque, (MEJRI 1997). Les séquences chaussure pour femme et patte-de-lièvre ${ }^{6}$ illustrent cette opposition. Dans les cas de transparence sémantique, la signification globale des noms composés d'artefact est directement déductible de celle de leurs composants. La séquence centrifugeuse pour fruits et légumes est analysable à partir des éléments qui la compose ; le premier signifie 'appareil de tri', les quatre derniers 'opérant sur un végétal consommable'. Dans les cas d’opacité sémantique, aucun constituant des noms d'artefact ne participe à sa compréhension globale. La séquence nid d'ange est inanalysable à partir des éléments qui la compose; ni nid ni d'ange ne permette d'accéder à sa signification : 'vêtement chaud très long, fermé en bas dans lequel on enveloppe un bébé'. De plus, les 
séquences sémantiquement opaques sont souvent polysémiques; par exemple, pied-de-biche a sept acceptions selon le Larousse L $^{7}$

La dichotomie opacité/transparence n’est pas toujours pertinente pour les noms d'artefact car il y a de nombreuses situations où les constituants des noms composés permettent partiellement ou indirectement d'accéder à leur signification. Ainsi, la signification 'véhicule' de traction avant n'est pas directement déductible à partir des mots traction et avant, mais la combinaison de leur signification implique un mode de déplacement et la façon dont ce mode de déplacement opère qui renvoie à un véhicule. L'étude des noms composés d'artefact fait ressortir que la plupart d'entre eux sont totalement, partiellement ou indirectement transparents sur le plan sémantique.

L'analyse des structures internes en termes de prédicat et d'argument conduit à classer les noms composés d'artefact d'une quatrième façon :

- $\quad$ argument prédicat : appareil photo ; porte de sortie, griffe à cintrer, bouton-poussoir, ...

- classe prédicat argument : porte-ski ; dégivreur de lunette, ...

- $\quad$ argument argument : mâchoire de frein, couteau-steak, ...

\subsection{Combinaison taxinomique}

Le croisement des différentes classifications des noms d'artefact permet d'expliquer leur fonctionnement sémantique. Les principaux cas de figure sont les suivants :

1. Les composés endocentriques, transparents, formés d'un argument suivi d'un prédicat permettent le plus souvent d'accéder directement à leur signification globale. Ils ont des configurations du type NOM+PREPOSITION+VERBE (machine à écrire), du type NOM+PREPOSITION+NOM (fer de calfatage) et du type NOM+NOM (crochet pétrisseur). Les constituant ont des propriétés sémantiques en rapport avec leur positionnement: les antéposés spécifient la nature des artefacts et les postposés leur finalité. Les postposés peuvent également signifier une propriété, comme en soie dans chemise en soie.

2. Les composés endocentriques, transparents et formés de deux arguments ont également une signification globale directement accessible à partir de celles de leurs constituants. Leurs configurations sont principalement du type NOM+NOM (piston-écrou) et du type NOM+PREPOSITION+NOM (porte de placard). La nature des relations entre les deux constituants explique les caractéristiques sémantiques de leur association. Il s’agit généralement d'une relation 'partie-tout' telle que le premier constituant dénote un élément du second: rondelle cuvette. Une relation 'tout-partie' est également possible : placard penderie. Il y a aussi d’autres relations, par exemple celle entre un objet et ce sur quoi il s’applique : fer à cheveux.

3. Les composés exocentriques, transparents et formés d'un prédicat suivi d’un argument ne permettent qu'indirectement d'accéder à leur signification globale. Leur configuration est du type VERBE+NOM (porte-chéquier). Les propriétés sémantiques des constituant définissent la fonctionnalité (prédicat antéposé) et ce sur quoi elle porte (argument positionnement). Leur dénomination est sans rapport avec celles des unités lexicales qui les composent.

4. Les composés exocentriques opaques ont une signification globale inaccessible à partir de leurs constituants, quels qu'ils soient: duchesse brisée. ${ }^{8}$ Ils peuvent avoir toutes sortes de configurations mais s'avèrent peu fréquents au sein des artefacts. Leur opacité sémantique 
explique l'absence de points commun entre leur dénomination et celle de leurs principaux constituants.

\subsection{Conséquences théoriques}

Il ressort de l'analyse de la structure interne des noms composés d'artefact que les noms argumentaux, d'une façon générale, seraient des matrices prédicatives. À ce titre, ils serviraient de point d'ancrage à des prédications dont les formes primitives seraient les énoncés préconstruits associés à chaque nom. Ces énoncés sont des savoirs langagiers que l'on peut concevoir comme autant de définitions des substantifs, faisant état des principaux attributs des objets qu’ils dénotent. Pour les noms d'artefact, les définitions portent principalement sur la fonctionnalité inhérente de ces objets ${ }^{9}$. Elles sont tributaires d'autre sortes de savoirs sur la langue, les dénominations. Ces dernières sont autant de présupposés qui s’intègrent aux énoncés préconstruits, $c f$. supra. Ces deux types de savoirs permettraient de produire ou d'interpréter des discours en rapport avec les référents des noms argumentaux.

Ainsi, pour les noms d’artefact, le substantif four à micro-onde, en tant qu'appareil dédié à la cuisson est associé à des énoncés préconstruits comme : Un four à micro-onde, cela cuit des aliments ; Un four à micro-onde cela grille des aliments ; Un four à micro-ondes, cela décongèle des aliments ; Un four à micro-onde, cela fait fondre des aliments ; Un four à micro-onde, cela mitonne des aliments ; Un four à micro-onde, cela réchauffe des aliments; etc. Ces formulations stéréotypiques mettent en avant des prédications en rapport avec la finalité de l'appareil qu'elles décrivent, à savoir 'cuisson'. Parce qu'elles le contiennent en puissance, les formulations expliqueraient la production et la compréhension de l'énoncé authentique suivant: J'utilise mon four micro-ondes pour réchauffer et décongeler des plats mais aussi cuire une confiture, faire fondre du chocolat, gratiner les aliments après la cuisson et cuire et rôtir toutes sortes de viandes.

L’objet d'étude pris en compte ici fait ressortir le rôle prépondérant des noms argumentaux en langue du fait qu'ils sont potentiellement porteurs des énoncés qui les impliquent. Dans la mesure où la fonction argumentale s'applique, par définition, aux noms argumentaux, elle aurait donc un rôle de premier plan dans le modèle de données. Cette analyse implique de réviser son positionnement par rapport à la fonction prédicative car, au lieu d'être subordonnée à cette dernière, elle l’incorporerait.

\section{Conclusion}

La théorie des trois fonctions primaires est fondée sur une série de postulats. La conception de l'univers découle de la capacité à discriminer et à catégoriser des entités (postulat 1). Les représentations langagières des entités correspondent à des arguments au niveau logico-sémantique (postulat 2). A ce titre, ils sont impliqués dans les opérations de dénomination et sont pourvus d'une charge référentielle non négligeable (postulat 3). C'est pourquoi le mode d'organisation des arguments s'inscrit naturellement dans une perspective ontologique en termes de hiérarchisation et d'inclusion. Par ailleurs, ils sont organisés en fonction des prédications potentielles qu'ils autorisent. Celles-ci portent sur les propriétés des entités qu'ils dénomment et leurs relations. Aussi, les arguments se conçoivent également comme des matrices prédicatives produisant l'incorporation des arguments dans les prédicats sous une forme schématique (postulat 4).

Les autres postulats se rapportent au fonctionnement des prédicats et des actualisateurs. Ce qui importe ici, c’est de souligner le rôle premier des arguments dans la théorie. Le bien-fondé du modèle de données est en cours de validation. Les résultats de l'étude des noms d'artefact y participent en montrant en quoi leur spécificité sémantique se manifeste en termes de dénomination et de prédication. Des études portant sur d'autres sortes de noms argumentaux restent à faire pour mieux expliquer comment se positionne la 
fonction argumentale par rapport à la fonction prédicative. De même, des études approfondies des arguments phrastiques doivent contribuer à mettre évidence l'articulation entre la fonction argumentale et la fonction modélisatrice.

\section{Références bibliographiques}

ANSCOMBRE J.-C 2001, "Les syntagmes les N/des N en position sujet ou objet : un syntagme générique ou pas ? ", Lingvisticae Investigationes Supplementa 23, John Benjamins, Amsterdam.

BENVENISTE E 1966, Problèmes de linguistique générale 1, Gallimard, Paris.

BUVET P.-A. 2013, "Quelles procédures d'étiquetage pour la gestion de l'information textuelle électronique ?", L'information grammaticale 122, Éditions Peeters, Louvain.

BUVET P.-A. 2013, La dimension lexicale de la détermination, Champion, Paris.

CORBIN D. et P. CORBIN 1991, "Un traitement unifié du suffixe -ier(e) ", Lexique 10, Presses du Septentrion.

DALADIER A.1996, "Le rôle des verbes supports dans un système de conjugaison nominale et l'existence d'une voix nominale en français", Langages 121, Larousse, Paris.

DUBOIS J. 1962 : Étude sur la dérivation suffixale en français moderne et contemporain, Larousse, Paris.

FRADIN B. 1984, « Anaphorisation et stéréotypes nominaux », Lingua,64:4, Elsevier, Amsterdam.

GONZÁLEZ REY I. 2002, La phraséologie du français, Presses Universitaires du Mirail, Toulouse.

GUIRAUD P. 1967, La syntaxe du français, Presses Universitaires de France, Paris.

HARRIS Z. S. 1976, Notes du cours de syntaxe, Le Seuil, Paris.

HOEY M. 2005, Lexical Priming: A New Theory of Words and Language, Routledge, London and New-York.

IMBS P. 1960, "Au seuil de la lexicographie", Cahier de lexicologie 2, Didier, Paris.

KLEIBER G. 1981, Problèmes de référence : descriptions définies et noms propres, Klincksieck, Paris.

KLEIBER G. 1984, Dénomination et relations dénominatives, Langages, 76, Larousse, Paris.

KLEIBER G. 1990, L’article LE générique. La généricité sur le mode massif, Droz Genève-Paris.

KLEIBER G. et C. SCHNEDECKER, A. THEISSEN 2006, La relation partie-tout, Peters Publishers, Louvain.

LE GOFFIC P. 1993, Grammaire de la phrase française, Hachette, Paris.

LERAT P 1995, Les langues spécialisées, PUF, Paris.

MARTIN R. 2014, Sur les universaux du langage, Académie des inscriptions et belles lettres.

MATHIEU-COLAS M. et D. LE PESANT 1998, "Introduction aux classes d’objets", Langages, 131, Larousse, Paris.

MEJRI S. 1997, Le figement lexical : descriptions linguistiques et structuration sémantique, Faculté des lettres de la Manouba, Tunis.

HAJOK A. et S. MEJRI (dir.) 2011, Le figement linguistique et les trois fonctions primaires (prédicats, arguments, actualisateurs), Neophilologica, Volume 2", Université de Catowice.

MITTERAND H. 1963, Les mots français, PUF, Paris.

MORTUREUX M.-F.1984, "La dénomination, approche sociolinguistique", Langages 19, Larousse., Paris

PETIT G. 2009, La dénomination : approches linguistique et terminologique, Peeters, Leuven. 
POLGUERE A. 2003, Lexicologie et sémantique lexicale. Notions fondamentales, Les Presses de l'Université de Montréal, Montréal.

POLGUERE A. 2012, "Propriétés sémantiques et combinatoires des quasi-prédicats sémantiques", Scolia 26, Université des Sciences Humaines de Strasbourg.

PRUVOST J. 2006, Les Dictionnaires français, outils d'une langue et d'une culture, Ophrys, Paris-Gap.

PUSTEJOVSKY J. 1995, Generative Lexicon, Cambridge, Mass., MIT.

QUEMADA, B. 1984, Datations et documents lexicographiques, Matériaux pour l'histoire du vocabulaire français, 2e série, vol. 2, Klincksieck, Paris.

REYT A. 1977, Le Lexique : Images et modèles, Armand Colin, Paris.

REY-DEBOVE J. 1978, Le Métalangage : étude du discours sur le langage, Armand Colin, Paris

SLIWA D. 2000, Aspects dénominatifs de la morphologie dérivationnelle : étude des noms d'artefacts en français et en polonais, Red Wydawn, Katolickiego Uniwersytetu Lubelskiego.

\footnotetext{
${ }^{1}$ Les dictionnaires sont des ressources linguistes dédiés au traitement de l'information textuel. Leur microstructure exige une stricte homogénéité pour que les programmes informatiques puissent les prendre en compte.

${ }^{2}$ La relation de synonymie est également présente dans la description d’une entrée lexicale. Elle relève cependant de la glose et non de la définition, REYT 1977.

${ }^{3}$ Nous ne tenons pas compte ici des composés savants comme stéthoscope.

${ }^{4}$ Certains noms endocentriques sont atypiques ; par exemple, elle ne s'applique pas à la séquence aiguille-couteau implique à la fois aiguille et couteau.

${ }^{5}$ Pièce d'une aiguille ferroviaire assurant la jonction entre les rails concourants et les lames d'aiguille.

${ }^{6}$ Le nom composé patte-de-lièvre est un terme du domaine des transports signifiant 'courte section de contre-rail', un contre-rail étant unrail placé parallèlement à un autre rail à l'intérieur de la voie (source : WIKIPEDIA)

7 'poignée de sonnette figurant un pied de biche' ; 'petit levier métallique à la tête en biais, aplatie et fendue, qui sert à arracher les clous' ; 'pièce de la machine à coudre qui maintient le matériau à plat sur la platine et dont l'extrémité comprend deux branches entre lesquelles passe l'aiguille entraînant le fil de dessus' ; 'levier articulé à deux pinces, qui servait à tendre l'arc des arbalètes' ; 'levier métallique allongé, ayant une tête en biais et permettant de soulever de lourds fardeaux' ; 'pièce de bois dur entaillée d'un bout, servant à tenir le chant à travailler le long de l'établi' ; 'pied de meuble galbé, se terminant par un sabot'.

${ }^{8}$ Une duchesse brisée est une chaise longue divisée en deux ou trois parties distinctes et séparables. Le siège luimême est une bergère, prolongée soit par un fauteuil (ou un pouf) à l'assise allongée lui faisant face, soit par un pouf et un fauteuil faisant face (source : WIKIPEDIA).

${ }^{9}$ La finalité inhérente des référents des noms d'arfertact se manifeste sur le plan lexical en termes de fonction télique (PUSTEJOVSKY 1995).
} 